\title{
Comparative analysis of the $14-3-3$ gene and its expression in Echinococcus granulosus and Echinococcus multilocularis metacestodes
}

\author{
M. SILES-LUCAS*, C. P. NUNES and A. ZAHA \\ Centro de Biotecnologia, Universidade Federal do Rio Grande do Sul, Av. Bento Gonçalves 9500, \\ 9150-970 Porto Alegre, Brazil
}

(Received 11 May 2000; revised 7 September 2000; accepted 7 September 2000)

\begin{abstract}
SUMMAR Y
It was suggested that the unlimited proliferative capacity of the Echinococcus multilocularis metacestode may be related to overproduction of the 14-3-3 protein. As is known, the proliferative capacities of E. granulosus and E. multilocularis metacestodes are very different. By comparing the expression levels of the 14-3-3 gene between in vitro-obtained $E$. granulosus and E. multilocularis metacestodes, we were able to provide experimental evidence of the potential relation between 14-3-3 over-expression and tumour-like growth in E. multilocularis metacestodes. RT-PCR and Northern blot experiments indicated that 14-3-3 expression level is about 4-fold higher in the E. multilocularis metacestode. This differential expression was confirmed both by immunoblotting and immunocytochemistry experiments, which allowed detection of the protein in the cyst wall from E. multilocularis but not in the cyst wall from E. granulosus. The alignment of the Echinococcus 14-3-3 cDNA sequence with known 14-3-3 isoforms from other organisms, grouped the parasite sequence into the tumour growth-related isoforms. $†$ The known relation between over-expression of some 14-3-3 isoforms and tumour-related processes, together with the present results, suggest that the Echinococcus 14-3-3 protein could be one of the molecules responsible for the differences between E. granulosus and E. multilocularis metacestode growth behaviour.
\end{abstract}

Key words: Echinococcus granulosus, Echinococcus multilocularis, metacestode, 14-3-3 gene, 14-3-3 protein, expression level.

\section{IN TRODUCTION}

The two best-known Echinococcus species, E. granulosus and E. multilocularis, produce unilocular (UE) and alveolar (AE) echinococcosis, caused by infection with the metacestode (larval) stage of the parasite. The differences between E. granulosus and $E$. multilocularis regarding their metacestode development is a well-known phenomenon (Thompson \& Lymbery, 1995). The metacestode of E. granulosus is typically unilocular, fluid-filled, consisting of a germinal layer supported externally by a non-cellular laminated layer and surrounded by a host-produced fibrous adventitial layer. In contrast, the metacestode of $E$. multilocularis is a multivesicular, infiltrating structure with no limiting host-tissue barrier, forming a network of filamentous cellular protrusions of the germinal layer, which are responsible for the tumour-like growth of the parasite in human patients.

* Corresponding author (present address): Institute of Parasitology, University of Berne, Länggass-Strasse 122, 3001-Berne, Switzerland. Tel.: +41 31 6312396. Fax: +41316312622. E-mail: siles@ipa.unibe.ch

$\uparrow$ Nucleotide sequence data reported in this paper are available in the EMBL, GenBank ${ }^{\text {(im }}$ and DDJB databases under the accession numbers AF207904 and AF207905.
$\mathrm{UE}$ and $\mathrm{AE}$ are severe parasitic diseases in man. Thus, major efforts have been made towards the improvement of diagnosis, prevention and treatment of echinococcal disease. In the last few years, efforts have been focused in several molecular approaches, some of them studying the developmentally regulated Echinococcus genes, as those concerning the stage-specifically regulated $14-3-3$ gene in E. multilocularis (Siles-Lucas et al. 1998). 14-3-3 genes have been related with aberrant cellular activity, as the corresponding proteins regulate the activity of other molecules involved in abnormal cell proliferation (e.g. Pallas et al. 1994). Thus, we have suggested a possible relationship between 14-3-3 over-expression and the unlimited growth of the $E$. multilocularis metacestode (Siles-Lucas et al. 1998). Based on the above-mentioned differences in the growing behaviour between $E$. granulosus and $E$. multilocularis cysts, we decided to compare the expression level of the 14-3-3 gene in the metacestodes from those two species. Our results demonstrate that, compared to E. granulosus metacestode, the 14-3-3 protein is over-expressed in the metacestode of $E$. multilocularis. Furthermore, the corresponding native protein could not be detected in the germinal layer of $E$. granulosus cysts. In addition, the alignment of the Echinococcus 14-3-3 protein sequence with different 
14-3-3 proteins from other organisms allows including the parasite protein into the tumour growing-related 14-3-3 group. An actual co-operational role of the over-expressed 14-3-3 molecule in the tumour-like growth capacity of $E$. multilocularis metacestode is suggested.

\section{MATERIALS AND METHODS}

\section{Parasites}

Echinococcus granulosus metacestode tissue (cyst wall and protoscoleces) was collected from a fertile lung cyst (isolate CW1), obtained from a naturally infected cow from a local abattoir as described elsewhere (Felleisen \& Gottstein, 1993).

\section{In vitro culture}

Microcysts of E. granulosus were obtained by in vitro cultivation of 50000 protoscoleces (isolate CW1, see above). The parasite was cultured basically as described before (Casado \& Rodriguez-Caabeiro, 1989; Hemphill \& Gottstein, 1995). Cysts were collected at day 90 of culture.

\section{Parasite extracts, SDS-PAGE and immunoblotting}

Somatic protein (S) extracts from E. granulosus protoscoleces and $\mathrm{S}$ extracts from $E$. granulosus cyst wall proteins, and total RNA from in vitro-obtained microcysts, were prepared as previously described (Siles-Lucas et al. 1998). $\mathrm{S}$ extracts from $E$. multilocularis protoscoleces and $\mathrm{S}$ extracts and RNA of cyst wall from in vitro recovered $E$. multilocularis microcysts (isolate Faub), obtained as described elsewhere (Casado \& Rodriguez-Caabeiro, 1989; Hemphill \& Gottstein, 1995), were a kind gift from Drs B. Gottstein, R. Felleisen and A. Hemphill (Institute of Parasitology, University of Berne, Switzerland). Methods for the extraction of proteins and RNA were performed as described elsewhere (Siles-Lucas et al. 1998). Following determination of protein concentration (Bradford, 1976), equal amounts of the above-mentioned protein extracts were separated in $12 \%$ SDS-PAGE under reducing conditions. After transfer of proteins onto nitrocellulose membranes (Gibco), immunoblot analyses were performed as described elsewhere (Siles-Lucas et al. 1998).

Reverse transcription $(R T)$, polymerase chain reaction $(P C R)$ and sequence analysis

A PCR-based approach was employed for amplification of the cDNA encoding the E. granulosus 14-3-3 protein. For amplification of the sequence between bases 58 and 909, total RNA from in vitro-obtained E. granulosus microcysts was reversely transcribed into single-stranded cDNA as described elsewhere (Felleisen \& Gottstein, 1994). The resulting cDNA was amplified as previously described (Siles-Lucas $e t$ al. 1998) using the gene-specific primers $1433 \cdot 1\left(5^{\prime}-\right.$ $\mathrm{GC}(\mathrm{C} / \mathrm{T}) \mathrm{AA}(\mathrm{A} / \mathrm{G}) \mathrm{C}$ T'TGC $(\mathrm{C} / \mathrm{T}) \mathrm{GA}(\mathrm{A} / \mathrm{G}) \mathrm{CA}-$ (A/G)GC) and 1433.2 (5'-C'TCAATCAGAACCACGACAG), or the primer 1433.3 (5'-GAGAAAATTGGTGCTGAAGC) in combination with an oligo-dT primer (dT16mer, Pharmacia). The sequence of the $5^{\prime}$-end of the cDNA encoding $E$. granulosus 14-3-3 (bases 1-365) was determined by sequencing of the PCR product obtained by amplification of $1 \mu \mathrm{l}$ ( $\sim 10^{7}$ plaque-forming units) of a $\lambda \mathrm{gt} 11$ E. granulosus protoscoleces cDNA library as template with the $\lambda$ gt11 forward (Gibco) and the $1433 \cdot 4$ (5' GCTTCAGCACCAATTTTC'TC) primers. The 1433 primers were designed based on of the known E. multilocularis 14-3-3 cDNA sequence (accession no. U63643). PCR products were sequenced with the Sequenase sequencing kit (Pharmacia) with respective primers. The same procedure was followed for confirmation of the E. multilocularis 14-3-3 cDNA sequence. Both amino acid sequences were compared between them and with the data-bank available protein sequences from the $\zeta$ (accession nos D83037, D87660, AW517476, AF033312, AA858662 and $\mathrm{P} 29361), \quad \beta$ (accession nos AF043736, P29358, AW518256, D17446 and $\mathrm{AAC} 14343$ ) and $\sigma$ (accession nos AAF36093, AAC52030 and P31947) 143-3 isoforms. Grouping of the Echinococcus 14-3-3 protein in the most related cluster was achieved by alignment of the active centres of the different 14-33 proteins (described by Siles-Lucas et al. 1998) using the CLUSTAL ${ }^{\text {IM }}$ computer package, and constructing the corresponding dendrogram with the Treeview ${ }^{\mathbb{I M}}$ computer program.

For the amplification of a partial exon from the EgactII E. granulosus actin gene (Da Silva et al. $1993)$, the same cDNAs were amplified as previously described (Siles-Lucas et al. 1998) with the Act1 (5'AC(C/T)AATTG(A/G)GATGA(C/T)ATG) and Act2 (5'-AAGCG(T/A)TC(G/C)TTGCC(A/G)ATG) primers, derived from the EgactII cDNA sequence (accession no. L07774). The corresponding PCR products-Egac and Emac-were cloned into the pMOS (Amersham) vector. The resulting subcloned products were used for transformation of Escherichia coli XL1B competent cells. After selection of recombinants and extraction of plasmid DNA, inserts were sequenced with the Sequenase sequencing kit (Pharmacia), with the $\mathrm{T} 7$ primer (Gibco).

\section{Semi-quantitative RT-PCR and Northern blot}

Total RNA from in vitro-cultivated E. granulosus and E. multilocularis microcysts was used for the comparison of the 14-3-3 gene expression levels between both metacestode tissues (germinal layer). 
A Em MSRLAECTISDTVDELVQRAKLAEQAERYDDMAGACKTMAEMGNELNNEERNLLSVAYKNVLGARRSSWR

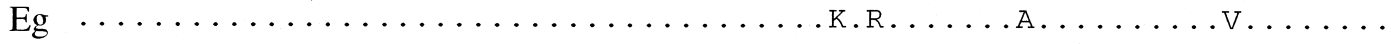

IMSSIAKKQAGTPLADQTDIYLKKVEEELIQICNDVLALPVLPITEKIGAEAKIFYYKMMGDYYRYLAEV

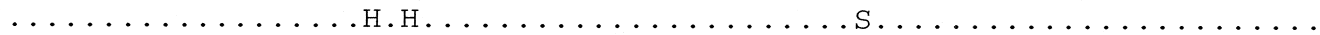

QEGEQNDKSTEAAEEANQKATSLAEAELSVTHPIRLGLALNF SVFYYEIKNMPEKACSLAKAAFDAAITE

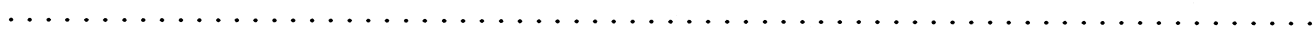

VDSIKDETYKDSTLIMQLLRDNLTLWNSECETDS

B
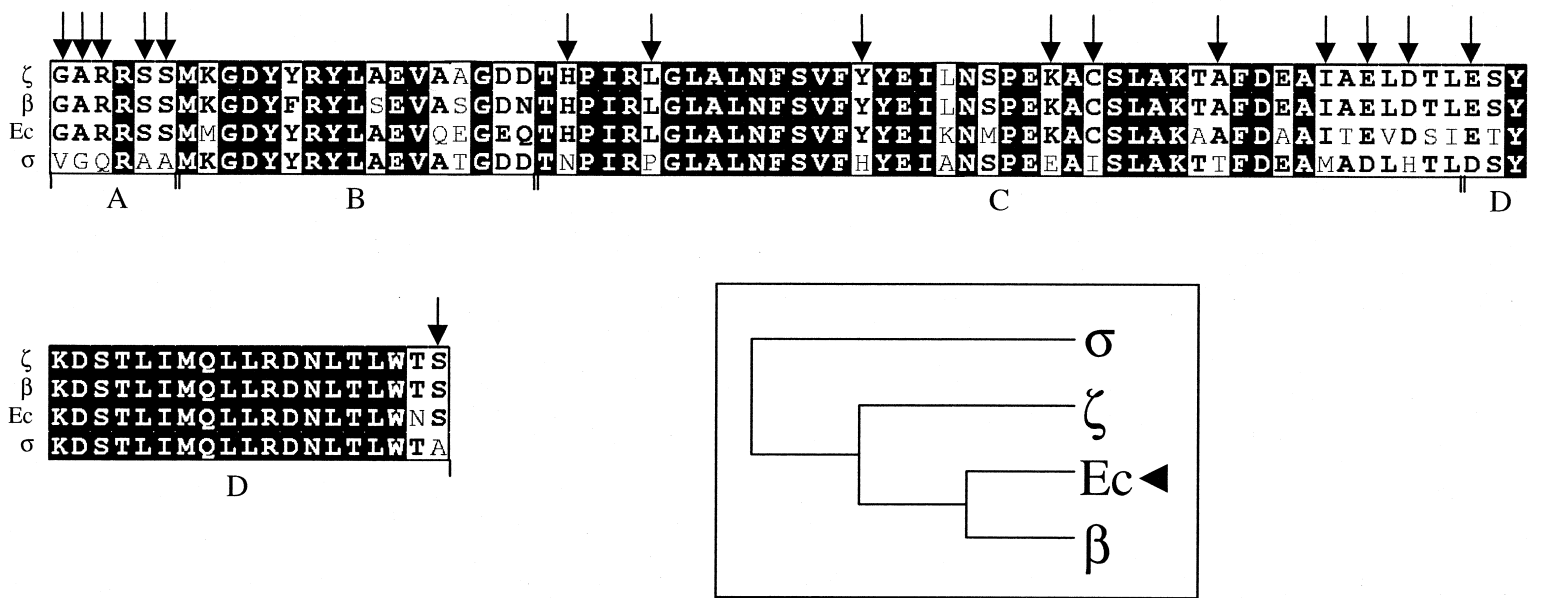

Fig. 1. (A) Aligned amino acid sequences of the Echinococcus multilocularis (Em, accession number U63643) and E. granulosus (Eg) 14-3-3 proteins. Dots indicate identity between both sequences. (B) The consensus sequence (Ec) from the active centres (A, protein kinase $\mathrm{C}$ pseudosubstrate; $\mathrm{B}$, carboxylic terminus of annexins; C, interaction site with Ras; D, 14-3-3 feature, as described by Siles-Lucas et al. 1998) of both parasite sequences where aligned and genetically compared with the corresponding consensus sequences of group 1 ( $\zeta$ and $\beta 14-3-3$ isoforms) and 2 ( $\sigma 14-$ 3-3 isoforms; see accession numbers in the Materials and Methods section). Homologous positions in the Echinococcus, $\zeta$ and $\beta$ isoforms, but different from $\sigma$ isoforms, are marked with arrows. Homologous positions in all sequences are represented in white, black-background letters. The arrowhead in the corresponding dendrogram shows the genetic position of the Echinococcus 14-3-3 sequence, clustered into group 1.

After measurement of their concentration by spectrophotometry, RNAs were reversely transcribed into single-stranded cDNA as previously described (Felleisen \& Gottstein, 1994). Following estimation of the cDNA concentration in each sample, the cDNAs were amplified as described before (SilesLucas et al. 1998) with the 1433.2 (above-mentioned) and 1433.5 (5'-ACATTTACCT'TAAGAAGGTG) primers, annealing at fully homologous positions in the E. granulosus and E. multilocularis 14-3-3 cDNAs. The corresponding internal control (from the constitutively expressed actinII gene) products were obtained by amplification of the abovementioned cDNAs, as described by Siles-Lucas et al. (1998), with the fully E. granulosus and E. multilocularis homologous Act3 (5'-ACCTTCTACAACGAACTACG) and Act4 (5'-ATCTGGCAGTTCATAAGAGC) primers, designed after sequencing of the Egac and Emac PCR products (see above). cDNA serial dilutions were used in parallel as templates for both PCR reactions in order to determine the respective cut-off points. The amount of 14-3-3 transcripts in the different samples could thus be compared in relation to the respective actinspecific cut-off point. The same total RNAs were used for Northern blot analysis as previously described (Siles-Lucas et al. 1998). The specific DNA probes for the Echinococcus 14-3-3 and EgactII genes were prepared by random priming of the corresponding affinity purified RT-PCR-specific products described above. Comparative quantitation of 14-3-3 and EgactII PCR-specific products or specific mRNAs from E. granulosus and E. multilocularis microcysts was done by densitometry, with the Kodak-Image ${ }^{(\mathbb{M})}$ computer program.

\section{Production of recombinant proteins}

The Eg14t and Em14t peptides, corresponding to the N-terminally truncated $E$. granulosus and $E$. multilocularis 14-3-3 proteins, respectively, were obtained as described before (Siles-Lucas et al. 2000). After purity checking by SDS-PAGE, recombinant proteins were used for immunization of 

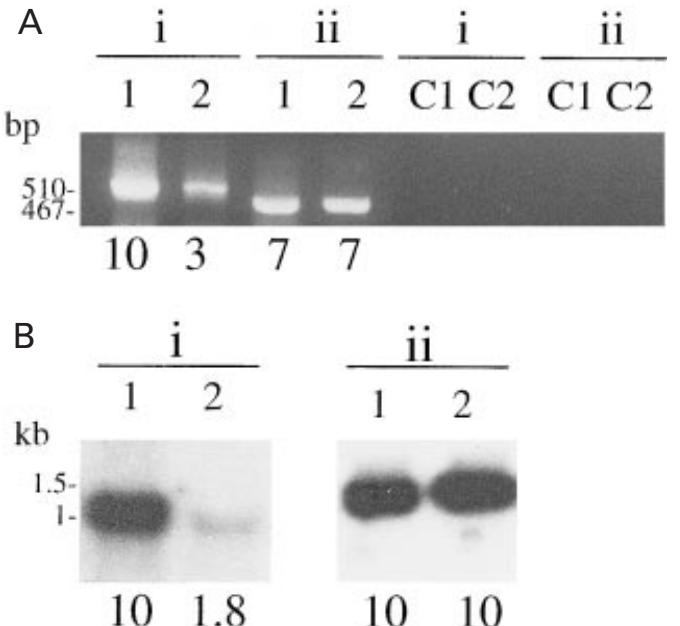

$10 \quad 1.8$

1010
Fig. 2. (A) Semi-quantitative RT-PCR (i) of the 14-3-3 and (ii) of the constitutively expressed EgactII genes of transcripts derived from RNA of (1) Echinococcus multilocularis and (2) E. granulosus in vitro-obtained microcysts. $\mathrm{C} 1$ and $\mathrm{C} 2$ are negative controls of the amplification reaction with non-reversely-transcribed RNAs and without DNA template, respectively. Fragment sizes are indicated in base pairs (bp). (2b) Northern hybridization of total (1) E. multilocularis and (2) E. granulosus RNAs probed with (i) $14-3-3$ and (ii) actin gene-specific fragments (see Materials and Methods section). Transcript sizes are indicated in kilobases $(\mathrm{kb})$. Intensity of bands, calculated by densitometry, are indicated as numbers at the bottom of each band.

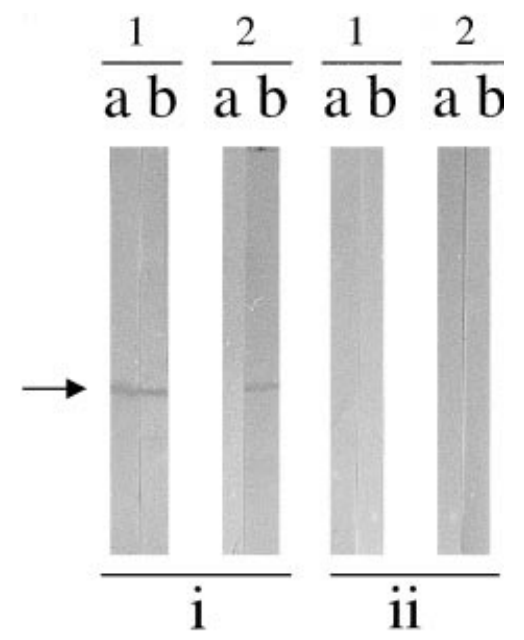

Fig. 3. Detection by immunoblot of the native 14-3-3 protein (marked with an arrow) in (a) cyst wall and (b) protoscoleces from (1) Echinococcus multilocularis and (2) E. granulosus, after SDS-PAGE in $12 \%$ gels under reducing conditions. (i) Reactivity of the anti-Eg14t hyperimmune mouse serum and (ii) control reactivity of the corresponding pre-immune mouse serum against respective protein extracts. Equal amount of proteins was loaded for all samples.

laboratory animals, as described elsewhere (SilesLucas et al. 2000). Sera were used for detection of the native 14-3-3 protein in different parasite tissues.

\section{Immunohistochemistry}

Immunolocalization of the native 14-3-3 protein was attempted in cyst wall and protoscoleces from $E$. granulosus collected from a fertile cow lung cyst (isolate CW1, see above) in fixed, paraffin-embedded parasitic material. This material was serially sectioned at $10 \mu \mathrm{m}$, and treated sections (as described by Thompson, Dunsmore \& Hayton, 1979) were used for the immunological reaction with the anti-Eg14t mice sera, basically as described elsewhere (Rodrigues et al. 1997). Sections were also stained with haematoxylin-eosin, as described previously (Thompson et al. 1979).

\section{RESULTS}

Sequence analysis of the $\mathrm{E}$. granulosus and $\mathrm{E}$. multilocularis 14-3-3 $c D N A s$

Sequencing of the $E$. granulosus 14-3-3 cDNA revealed a continuous open reading frame of 244 amino acids (Fig. 1), in which the characteristic 143-3 family motifs were detected. The E. multilocularis 14-3-3 cDNA sequence (accession number U63643) was confirmed and updated. The overall identity between the E. granulosus and E. multilocularis amino acid sequences was $97 \cdot 5 \%$. Comparative analysis of the Echinococcus 14-3-3 sequence and different 14-3-3 isoforms showed a higher similarity of the parasite sequence with the $\zeta$ and $\beta$ isoforms, and a lower similarity with the $\sigma$ isoforms (Fig. 1).

\section{$R T-P C R$ and Northern blot comparative analyses}

R'T-PCR amplification of the EgactII gene produced the expected $467 \mathrm{bp}$ band in both E. granulosus and E. multilocularis metacestode reactions, with comparable intensity in both samples (Fig. 2). The 14-33 -specific amplification of the same samples gave the expected $510 \mathrm{bp}$ bands, being the intensity of the PCR product remarkably higher for the $E$. multilocularis cDNA sample. No amplification was detected when non-reversely transcribed RNA or when no template was added to the $\mathrm{PCR}$ reaction (Fig. 2). RT and $\mathrm{PCR}$ reactions were repeated at least 3 times. The corresponding PCR specific fragments from the 14-3-3 and actin genes where used as probes for Northern hybridization of the same RNAs used for RT-PCR. Both probes gave single labelled bands of approximately 1 (14-3-3) and $1 \cdot 5$ (actin) $\mathrm{kb}$ for both E. granulosus and E. multilocularis RNAs. The intensity of the actin-specific bands was comparable for both samples, while the 14-3-3 band was notably more intense for $E$. multilocularis than for $E$. granulosus metacestode RNAs (Fig. 2). On the basis of the RNA and cDNA concentrations used for RT-PCR, the RT-PCR reaction cut-off points, and the densitometry analysis of the corresponding PCR 

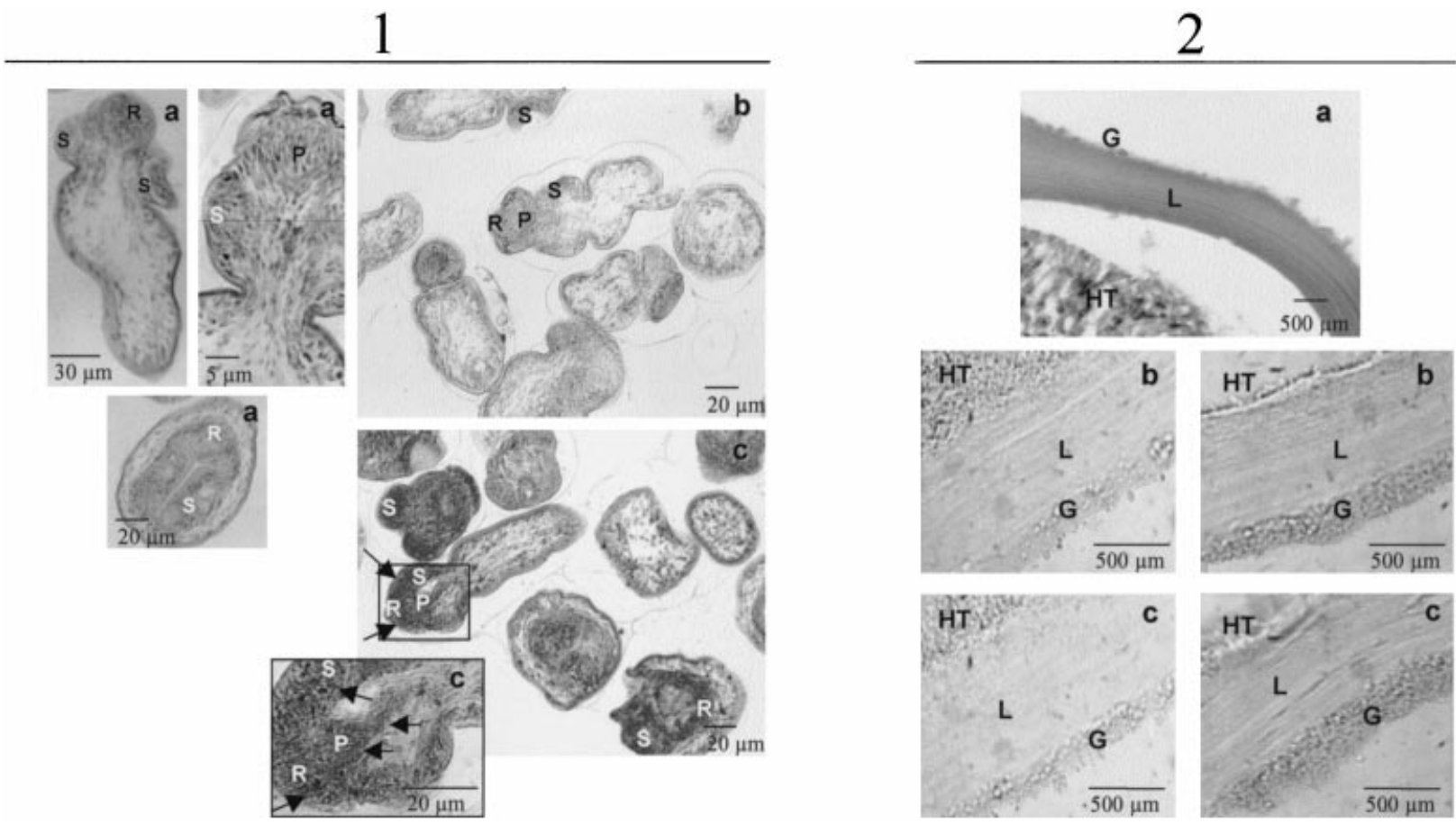

Fig. 4. Immunolocalization of the native 14-3-3 protein in Echinococcus granulosus (1) protoscoleces and (2) cyst wall. Sections were stained with (a) haematoxylin-eosin or probed with (b) a pre-immune or (c) the anti-Eg14t mouse serum. Anti-14-3-3 reactivity, marked with arrows, was detected in the pad and adjacent structures, and in the suckers (1c, see squared magnification in the bottom). No anti-14-3-3 reactivity was detected in germinative or laminated cyst layers (2c, two different sections). R, rostellum; P, pad; S, sucker; L, laminated layer; G, germinative layer; HT, host tissue.

bands and the transcripts detected by Northern blot, the expression level of the 14-3-3 gene in the metacestode of E. multilocularis can be estimated to be about 4 times higher than in the metacestode of E. granulosus.

\section{Immunoblotting}

Detection of the native Echinococcus 14-3-3 protein was achieved by immunoblot analysis with several hyperimmune anti-Echinococcus 14-3-3 mice sera. Under reducing conditions the native $14-3-3$ protein, with an apparent molecular mass of about $24.5 \mathrm{kDa}$, was detected on blots containing $E$. granulosus and $E$. multilocularis protoscoleces extracts, and on $E$. multilocularis cyst wall extracts (Fig. 3). The protein was not detected in E. granulosus cyst wall extracts (Fig. 3).

\section{Immunohistochemistry}

The detection of the 14-3-3 protein in sections of $E$. granulosus protoscoleces with the anti-Eg14t mouse serum 14a resulted in a strong reactivity localized in areas detected with the haematoxylin-eosin stain as those with the highest cellular density (Fig. 4). No positive reaction was observed in sections of $E$. granulosus cysts, neither in the laminated nor in the germinal layer, as shown in Fig. 4. Similar results were obtained with the $14 \mathrm{~b}$ to $14 \mathrm{e}$ anti-Eg14t mice sera (data not shown). No reactivity was detected when the corresponding pre-immune mice sera were used for the immunological reaction (Fig. 4).

\section{DISCUSSION}

Employing a PCR-based strategy, we sequenced the complete 14-3-3 E. granulosus cDNA. The comparative sequence analysis demonstrated its high homology (almost identity) with the corresponding gene in E. multilocularis (Siles-Lucas et al. 1998).

14-3-3 proteins have been repeatedly associated with tumour processes. This was frequently demonstrated by showing the interaction of 14-3-3 proteins with promoters of oncogenic events - e.g. the middletumour antigen (Pallas et al. 1994), the $\epsilon$ subtype of the protein kinase C (PKC $\epsilon$; Acs et al. 1995), the cMyc ('Tzivion, Luo \& Avruch, 1998) and the Raf-1 (McPherson et al. 1999). The activity of the abovementioned molecules depends on their interaction with 14-3-3 proteins and is enhanced when 14-3-3 proteins are over-expressed. Over-expression of 143-3 proteins has also been directly associated with lung tumours, being a monoclonal anti-14-3-3 antibody the election tool for the diagnosis of this disease in sputum samples (Nakanishi et al. 1997).

We have previously described that the 14-3-3 protein is over-expressed by the metacestode of $E$. multilocularis in comparison with the adult worm, and mainly localizes in the germinal layer of the cyst 
(Siles-Lucas et al. 1998). As mentioned, the $E$. multilocularis metacestode growth is characterized by the unlimited infiltration of its germinal layer within the host tissue. A possible role of the overexpression of the 14-3-3 protein in mediating the uncontrolled E. multilocularis (germinal layer) metacestode growth was thus suggested. The rate of the E. granulosus metacestode growth differs markedly when compared to that of the $E$. multilocularis metacestode. In E. granulosus, it is slow and limited by the host-derived adventitial layer (Thompson \& Lymbery, 1995). However, no experimental molecular-based difference related with these very distinct growth behaviours has been described so far. Thus, our aim was to gain an initial insight on the possible role of the level of 14-3-3 protein expression in promoting the uncontrolled growth of E. multilocularis cyst, when compared with the non-metastasic E. granulosus metacestode.

To avoid host influence in the parasite development (Thompson \& Lymbery, 1995), we obtained parasite material using an in vitro cultivation model (Casado \& Rodriguez-Caabeiro, 1989; Hemphill \& Gottstein, 1995). By a semiquantitative RT-PCR assay and Northern blot on total RNAs isolated from these in vitro obtained microcysts, we were able to detect a 4-fold 14-3-3 over-expression in the metacestode of E. multilocularis compared to E. granulosus. By immunoblotting, the native 14-3-3 protein could not be detected in cyst wall extracts of $E$. granulosus. On the contrary, a strong reactive band was found in the E. multilocularis cyst wall extracts. A band corresponding to the same size was also detected in protoscoleces extracts from both species. When detection of native protein was attempted on parasite sections, no signal showed up in laminated or germinal E. granulosus cyst layers, while the protein could be detected in protoscoleces from this species. As it is known, a second Echinococcus 14-33 isoform is produced by adult worms (Siles-Lucas et al. 2000). Thus, it remains to be clarified whether protoscoleces share one of the already characterized Echinococcus 14-3-3 isoforms or whether they possess their own. As it was previously demonstrated, the 14-3-3 protein can be readily visualized in the tumour-like proliferative E. multilocularis germinative layer (Siles-Lucas et al. 1998). These divergences in 14-3-3 expression and localization could in fact be related with the referred growth rate differences between both metacestodes.

As mentioned, 14-3-3 proteins have been associated with tumour processes. The $\sigma$ isoforms have been found to be down-regulated in several carcinoma types, or over-expressed after gamma irradiation of cells (e.g. Hermeking et al. 1997; Ostergaard et al. 1997). On the other hand, $\beta$ and $\zeta$ isoforms are remarkably over-expressed by tumour cells, and they have been related at molecular level with an extraordinary activation of the oncogenic
PKC $\epsilon$ protein when over-expressed (Acs et al. 1995; Setoguchi et al. 1995; Nakanishi et al. 1997). Other molecular relationships between over-expressed 143-3 proteins and different oncogenic molecules have been repeatedly demonstrated. Unfortunately, details about 14-3-3 isoforms involved in such interactions are mostly lacking. Trying to correlate further the 14-3-3 over-expression in E. multilocularis cysts and its tumour-growth behaviour, we aligned the active centres of the parasite protein sequence with the corresponding available amino acid sequences from the 'pro-tumourigenic' $(\beta$ and $\zeta$, called here 'group 1') and 'anti-tumourigenic' $(\sigma$, called here 'group 2') 14-3-3 isoforms. In the corresponding dendrogram, the Echinococcus 14-3-3 sequence is assigned to the group 1 ('pro-tumourigenic' isoforms).

In conclusion, we demonstrated the overexpression of the 14-3-3 protein in the tumour-like growing E. multilocularis metacestode, when compared with the slow-rate growing E. granulosus cysts. The over-expressed protein mainly localizes in the germinal layer of the parasite, which is in fact the structure responsible for the tumourigenic growth of the metacestode. The respective protein was not detectable in the germinal layer of the E. granulosus cysts. In addition, the parasite 14-3-3 protein could be genetically grouped with the 'pro-tumourigenic' 14-3-3 isoforms. Thus, we suggest the involvement of the over-expressed 14-3-3 protein in the promotion and/or maintenance of the progressive growth capacity of the larvae of E. multilocularis.

This work was supported by the Conselho Nacional de Desenvolvimento Científico e Tecnólogico, Brazil (Contract 300862/97-0 and Project PADCTII 62.0081/95.3). Authors would like to thank Drs B. Gottstein, R. Felleisen and A. Hemphill for providing E. multilocularis parasite extracts, and S. González and M. Marco for providing the paraffin-embedded parasite material. Thanks are given to Dr N. Müller for critical reading of the manuscript.

\section{REFERENCES}

ACS, P., SZAllasi, Z., KAZANieTZ, M. G. \& BlUMBerG, P. M. (1995). Differential activation of PKC isozymes by 143-3 zeta protein. Biochemical and Biophysical Research Communications 216, 103-106.

BRADFORD, M. M. (1976). A rapid and sensitive method for the quantitation of microgram quantities of protein utilizing the principle of protein-dye binding. Annals of Biochemistry 72, 248-254.

CASADO, N. \& RODRIGUEZ-CAABEIRO, F. (1989).

Ultrastructural study of in vitro larval development of Echinococcus granulosus protoscoleces. International

Fournal for Parasitology 19, 21-28.

DA SIlVA, C. M., FERREIRA, H. B., PICON, M., GORFINKEL, H., EHRLiCH, R. \& ZAHA, A. (1993). Molecular cloning and characterization of actin genes from Echinococcus granulosus. Molecular and Biochemical Parasitology 60, 209-219. 
FELleisen, R. \& GOTTSTEIN, B. (1993). Echinococcus multilocularis: molecular and immunochemical characterization of diagnostic antigen II/3-10. Parasitology 107, 335-342.

Felleisen, R. \& GotTstein, B. (1994). Comparative analysis of full-length antigen II/3 from Echinococcus multilocularis and E. granulosus. Parasitology 109, 223-232.

HeMPhill, A. \& GOTTSTEIn, B. (1995). Immunology and morphology studies on the proliferation of in vitro cultivated Echinococcus multilocularis metacestodes. Parasitology Research 81, 605-614.

Hermeking, H., Lengauer, C., POlyak, K., He, T. C., ZHANG, L., THIAGALINGAM, S., KINZLER, K. W. \& vOGELSTEIN, B. (1997). 14-3-3 sigma is a p53-regulated inhibitor of G2/M progression. Molecular Cell 1, 3-11.

LAEMMLI, U. K. (1970). Cleavage of structural proteins during the assembly of the head of bacteriophage $\mathrm{T} 4$. Nature 277, 680.

MCPHERSON, R. A., HARDING, A., ROY, S., LANE, A. \& HANCOCK, J. F. (1999). Interactions of c-Raf-1 with phosphatidylserine and 14-3-3. Oncogene 18, 3862-3869.

NAKANishi, K., HASHizUme, S., KATO, M., HONJOH, T., SETOGUCHI, y. \& YASUMOTO, K. (1997). Elevated expression levels of the 14-3-3 family of proteins in lung cancer tissues. Human Antibodies 8, 189-194.

OSTERGaARD, M., RASMUSSEN, H. H., NiELSEN, H. V., VORUM, H., ORNTOFT, T. F., WOLF, H. \& CELlis, J. E. (1997). Proteome profiling of bladder squamous cell carcinomas: identification of markers that define their degree of differentiation. Cancer Research 57, 4111-4117.
PAllas, D. C., FU, H., HAEHNEL, L. C., WEller, W., COLliER, R. J. \& ROBERTS, T. M. (1994). Association of polyomavirus middle tumor antigen with 14-3-3 proteins. Science 265, 535-537.

RODRIgUES, J. J. S., FERREIRA, H., FARIAS, S. \& ZAHA, A. (1997). A protein with a novel calcium-binding domain associated with calcareous corpuscles in Echinococcus granulosus. Biochemical and Biophysical Research Communications 237, 451-456.

SETOGUCHI, Y., KATO, M., SHOJI, M., HONJOH, T., KAMEI, M., SUgitani, M., SATo, s., Hashizume, s., HaNAgiri, T. \& yoshimatsu, T. (1995). Immunocytochemical detection of lung cancer cells with monoclonal antibodies to 14-3-3 proteins. Human Antibodies and Hybridomas 6, 137-144.

SILES-LUCAS, M., FELLEISEN, R. S., HEMPHILl, A., WILSON, W. \& GOTTSTEIN, B. (1998). Stage-specific expression of the 14-3-3 gene in Echinococcus multilocularis. Molecular and Biochemical Parasitology 91, 281-293.

SILES-LUCAS, M., NUNES, C. P., ZAHA, A. \& BREIJO, M. (2000). The 14-3-3 protein is secreted by the adult worm of Echinococcus granulosus. Parasite Immunology 22, 521-528.

THOMPSON, R. C. A., DUNSMORE, J. D. \& HAYTON, A. R. (1979). Echinococcus granulosus: Secretory activity of the rostellum of the adult cestode in situ in the dog. Experimental Parasitology 48, 144-163.

THOMPSON, R. C. A. \& LYMBERY, A. J. (1995). Echinococcus and Hydatid Disease. CAB International, Wallingford (UK).

TZIVION, G., LUO, Z. \& AVRUCH, J. (1998). A dimeric 14-33 protein is an essential cofactor for Raf kinase activity. Nature, London 394, 88-92. 